\title{
Reporte de caso: diabetes mellitus tipo 1 inducida por pembrolizumab en un paciente con adenocarcinoma de pulmón
}

\author{
Case report: pembrolizumab-induced Type 1 diabetes mellitus in a patient \\ with lung adenocarcinoma
}

Bedoya-Trujillo $L N^{1}$, Bedoya-Trujillo $P N^{2}$.

${ }^{1}$ Hematología y Oncología - Clínica Vida de Medellín.

${ }^{2}$ Estudiante de pregrado del programa de Biología, Facultad de Ciencias, Universidad del Tolima; pnabedoyatr@ut.edu.co.

Autor de correspondencia: Lady Nataly Bedoya Trujillo

Correo electrónico: mdnataly3821@gmail.com

Fecha de recepción: 13/09/2019

Fecha de aceptación: 14/03/2020

\section{Resumen}

Presentamos el caso de una paciente sin comorbilidades metabólicas con diagnóstico de adenocarcinoma de pulmón en manejo con pembrolizumab, quien, posterior al tercer ciclo de tratamiento, debuta con cetoacidosis diabética, requiere manejo para crisis hiperglucémica e insulina. Se realizó diagnóstico de diabetes mellitus tipo 1 y se reintrodujo el tratamiento de pembrolizumab a $200 \mathrm{mg}$ cada tres semanas además del manejo con insulina. En la actualidad la paciente permanece con una respuesta parcial posterior a 1 año del diagnóstico y sigue en manejo con insulinoterapia. En vista que en la actualidad no se cuenta con protocolos y guías de manejo para esta entidad, se realiza revisión del manejo y de la literatura debido a que solo se encuentran 6 casos reportados, siendo este el primer caso reportado en Colombia.

Palabras clave: neoplasias pulmonares, cetoacidosis diabética, diabetes mellitus tipo 1, insulina, anticuerpos monoclonales humanizados (anti-PD-1).

\section{Abstract}

We present a female patient without metabolic comorbidities, diagnosed with lung adenocarcinoma, in management with pembrolizumab after the third course of treatment, debuted with diabetic ketoacidosis, requiring management for hy- perglycemic crisis and insulin needed. Diabetes mellitus type 1 is diagnosed, to get control of this metabolic complication infrequent in immunotherapy, it is decided to reintroduce pembrolizumab treatment at $200 \mathrm{mg}$ every three weeks in addition to insulin management. At present the patient remains with a partial response after 1 year of diagnosis and continues to be managed with insulin therapy. Given that currently there are no protocols and management guidelines for this entity, a review of the management and the literature is carried out because only 6 reported cases are found, this being the first case reported in Colombia.

Keywords: Lung Neoplasms; Diabetic ketoacidosis; Diabetes mellitus type 1; Insulin; Humanized Monoclonal Antibodies (anti-PD-1).

\section{Introducción}

El pembrolizumab es un anticuerpo monoclonal tipo inmunoglobulina G4 isótopo kappa, con una alteración en la secuencia estabilizadora en la región del fragmento cristalizable (Fc), frente a la muerte celular programada 1 (PD-1); es decir, bloquea el ligando inhibidor del receptor de muerte celular programada 1 (PD-1) en las células $\mathrm{T}$ (1) producido en las células del hámster chino mediante tecnología de ácido desoxirribonucleico (ADN) recombinante (1). En este grupo de medicamentos también se encuentra el nivolumab y al atezolizumab, los cuales están aprobados para múltiples neoplasias y que han cambiado los resultados en oncología, como la sobrevida de libre progresión (PFS) y mortalidad, en patologías como melanoma, cáncer de pulmón y linfomas, aumentando también la sobrevida en enfermedad metastásica (2). Adicionalmente, estos anticuerpos han sido asociados a enfermedades autoinmunitarias como la neumonitis, la hepatitis, la nefritis y las endocrinopatías (3). 
La unión de PD-L1 y PD-1 evita que la célula T libere sus citocinas y destruya la célula con PD-L1 (4). Teniendo esto en cuenta, PD-1, como punto de control inhibitorio, se expresa sobre las células T, B, monocitos, tipo Natural Killer's (NK), dendríticas, macrófagos e islotes pancreáticos, siendo estos últimos una de las principales razones para el desarrollo de diabetes mellitus tipo 1 (5), pues las células T autorreactivas son los principales mediadores en la destrucción de las células beta pancreáticas (5).

Se ha reportado que los inhibidores de los puntos de control inmunológico, como pembrolizumab, pueden inducir efectos secundarios inmunomediados, incluida la diabetes mellitus tipo 1 en casos muy raros $(0,1 \%$ en ensayos clínicos) (6); otros autores reportan valores similares, como 0,2\% de los casos de los anticuerpos monoclonales como los anti-PD-1 (7). De acuerdo con la revisión de la literatura, actualmente existen 6 casos reportados en la literatura médica que aproximan el diagnóstico y el manejo (1). Es importante destacar que estos informes de casos se evaluaron teniendo en cuenta aspectos como la relevancia del título para la información reportada, la descripción adecuada del paciente (datos demográficos, anomalías de laboratorio y antecedentes médicos), y la descripción de la diabetes autoinmune/diabetes tipo 1 y su resultado (1). Por lo tanto, teniendo en cuenta estos aspectos, es posible realizar una posterior comparación entre estos informes y el caso presentado en el presente artículo.

\section{Presentación del caso}

Paciente femenina de 54 años, no fumadora, sin comorbilidades, quien en septiembre de 2017 desarrolló disnea progresiva, pérdida de peso, diaforesis y dolor en el hombro derecho. Se realizó tomografía computarizada de tórax (TC) donde se evidenció derrame pleural derecho y masa de 76 por $36 \mathrm{~mm}$ en el lóbulo superior ipsilateral y engrosamiento pleural de $35 \mathrm{~mm}$. Se realizó toracostomía cerrada, decorticación y pleurodesis con talco y se procedió a tomar muestras para estudio histopatológico que confirmaron cáncer de pulmón, tipo adenocarcinoma mal diferenciado, de patrón sólido con multicitoqueratina $\mathrm{AE} 1 / \mathrm{AE} 3$, factor de transcripción tiroidea 1 (TTF1) focal positivo, factor de crecimiento epidérmico (EGFRE) negativo, cinasa de linfoma anaplásico (ALK) negativo y PDL1 mayor al 50 \%. La estadificación evidenció compromiso metastásico pleural y óseo a nivel de la cabeza del húmero derecho y en múltiples arcos costales. La imagen de resonancia magnética cerebral con gadolinio (IRM) fue negativa y la TC de abdomen y pelvis fue normal, debido a que no se encontraron lesiones metastásicas en el sistema nervioso central.

Al examen clínico se encontró una funcionalidad por índice de Karnofsky (IK) del 70 \%, requerimiento permanente de oxígeno, disnea de mínimos esfuerzos y dolor 10/10 en el hombro derecho, el peso se encontraba dentro del rango nor- mal con un valor de IMC de 24,5. Se inició manejo paliativo con radioterapia antiálgica en dosis de 2000 centigray (cGy) en el hombro derecho y pembrolizumab cada 21 días. Después del tercer ciclo de inmunoterapia, la respuesta parcial en la tomografía de tórax fue reportada como engrosamiento pleural, atelectasias subsegmentarias en la base pulmonar derecha, algunas adenomegalias mediastinales perivasculares y paratraqueales y desaparición completa de la lesión del lóbulo superior derecho. Los paraclínicos evidenciaron hormona estimulante de la tiroides (TSH), tiroxina libre (T4), hemograma, función hepática, renal y glucosa normales. Clínicamente estable, sin requerimiento de oxígeno, sin disnea, auscultación pulmonar normal y un IK del $100 \%$ posterior al tercer ciclo, siendo cada ciclo de 21 días.

La paciente reinició sus actividades laborales al encontrarse completamente asintomática, sin embargo, previo al inicio del ciclo 4 de pembrolizumab, consultó por poliuria, polidipsia, polifagia, desorientación y deshidratación. Se encontró glucemia de $309 \mathrm{mg} / \mathrm{dL}$ (Tabla 1), gases arteriales con acidosis metabólica moderada, con una brecha aniónica mayor de 12 , cetonuria y cetonemia positivas, el hemograma y electrolitos fueron normales, la creatinina en $1,4 \mathrm{mg} / \mathrm{dL}$, valor normal (VN) de 0,5-1,0 mg/dL. Con estos resultados se diagnosticó cetoacidosis diabética y se inició hidratación e insulina endovenosa hasta alcanzar la normalización de los niveles de glucosa y estabilización clínica.

Tabla 1. Laboratorios

\begin{tabular}{l|c|c|c|c}
\hline Prueba & Ciclo 1 & Ciclo 2 & Ciclo 3 & Ciclo 4 \\
\hline TSH & 2,6 & 1,24 & 2,24 & \\
\hline Glucosa & $\begin{array}{c}102 \mathrm{mg} / \\
\mathrm{dL}\end{array}$ & $\begin{array}{c}100 \mathrm{mg} / \\
\mathrm{dL}\end{array}$ & $\begin{array}{c}309 \mathrm{mg} / \\
\mathrm{dL}\end{array}$ & $\begin{array}{c}128 \mathrm{mg} / \\
\mathrm{dL}\end{array}$ \\
\hline
\end{tabular}

Luego de 12 horas de manejo se ordenaron análogos de insulina como Lantus (glargina) y Apidra (glulisina), con dosis de $0,3 \mu \mathrm{g} / \mathrm{kg}$ por día y posterior ajuste según glucometrías y evaluación de endocrinología, con un ajuste de la dosis según requerimiento, con lo que se logró el alta de la paciente con esquema basal/bolo. Se tomó péptido C: 0,12 ng/mL VN (1,1-4,4 ng/mL); insulina libre: 2,38 (2,9-19); e insulina total: $0,26(6,0-27)$ por lo que se consideró una diabetes mellitus tipo 1. Una vez estabilizados los niveles de glucosa, se reinició tratamiento con pembrolizumab al siguiente ciclo, sin nuevas complicaciones. Actualmente ha completado 1 año de diagnóstico de cáncer de pulmón, y aún permanece con respuesta oncológica parcial según los criterios de respuesta en tumores sólidos (RECIST) 1.1 al manejo con inmunoterapia y con su diabetes mellitus controlada. 


\section{Discusión y revisión de la literatura}

Hoy día el manejo del cáncer está viviendo una revolución gracias a los resultados obtenidos con la inmunoterapia. El propósito de la inmunoterapia es iniciar o reiniciar y amplificar la inmunidad contra las células tumorales. En algunos tumores existe una desregulación del sistema inmunitario que causa alteración de la proliferación de las células T, disminución de la producción de citocinas y contribución a la inhibición de la vigilancia inmunitaria activa de los linfocitos T a los tumores, lo que explicaría la evasión del sistema inmunitario de los tumores y permitiendo su crecimiento neoplásico a pesar de tener una inmunidad aparentemente intacta (8). En términos generales, el PD-1 actúa como coinhibidor del receptor expresado sobre las células $\mathrm{T}$ activadas y oculta el tumor a los linfocitos T, lo que permite el desarrollo tumoral (9). El pembrolizumab bloquea su interacción con los ligandos PD-L1 Y PD-L2, lo que potencia la respuesta de las células T y activa la respuesta inmunitaria de manera específica mediante el bloqueo de PD-1, unido a PD-L1 y PD-L2, que se expresa en las células presentadoras de antígenos y que se puede expresar en tumores u otras células del microambiente tumoral (10).

Las toxicidades y los eventos adversos con inmunoterapia están relacionados con el incremento de las citocinas inflamatorias. Las más comunes son las dermatológicas tales como erupciones eritematosas maculopapulares o prurito (47 \%-68 \%) que se evidencian en las primeras semanas (11), diarrea (30\%$35 \%$ ), enterocolitis (5\%), perforación intestinal (menos del 1 $\%$ ), obstrucción intestinal, hepatotoxicidad (2 \%-9 \%) y endocrinopatías evidenciadas después de la tercera dosis, como hipofisitis (1,5\% [0\%-17\%]), tiroiditis autoinmunitaria, epiescleritis, uveítis y otras menos comunes, como pancreatitis (1,5\%), neumonitis, diabetes tipo 1 (0,2\%), entre otras (12).

La diabetes tipo 1 asociada a pembrolizumab está relacionada con la incapacidad de este medicamento para distinguir entre células normales y cancerígenas. Se cree que el mecanismo de acción es la producción de anticuerpos que destruyen las células de los islotes pancreáticos que son responsables de la regulación y la producción de la insulina (13). En modelos murinos y en los hallazgos histológicos de ratones que desarrollaron diabetes tipo 1 se encontró destrucción de las células en los islotes de Langerhans y también un nivel incrementado de expresión de PD-L1 en los islotes celulares (14). Esto muestra que el fármaco actúa directamente en los islotes pancreáticos beta y ayuda a la destrucción de la producción de insulina. La destrucción de los islotes beta produce una liberación de proteínas intracelulares, tales como la descarboxilasa de ácido glutámico (GAD) (15). Esta es una enzima que se encuentra en los islotes pancreáticos beta y desempeña un papel en la descarboxilación del glutamato a ácido gamma-aminobutírico (GABA) (16). Cuando esta proteína intracelular se libera, se activa el sis- tema inmunitario y forma anticuerpos anti-GAD, simulando un fenómeno patológico similar al evidenciado en la diabetes mellitus tipo 1, que lleva a estos pacientes a presentar cetoacidosis diabética (17). En cuanto al manejo con esteroides que son utilizados en otras complicaciones de la inmunoterapia no se ha evidenciado ningún beneficio en la aparición de diabetes tipo 1 por lo cual no se recomiendan (4). Se requieren más investigaciones acerca de la fisiopatología y de los posibles marcadores para prevenir y evitar esta complicación.

Los 6 casos reportados en la literatura, los cuales se presentan en comparación con el caso presentado en la Tabla 2, se caracterizaron porque no hubo predilección por sexo (tres hombres y tres mujeres afectados) ni con la edad de presentación (promedio de 58 años). Adicionalmente, la mayoría de los pacientes afectados tenía un diagnóstico inicial de melanoma a pesar de que no existe información suficiente para establecer una relación de significancia en cuanto al diagnóstico y las posteriores afectaciones. De manera similar al caso presentado en este informe, la mayoría de los casos se presentaron con cetoacidosis diabética (CAD) (4/6 casos), de los cuales todos se manejaron por fluidos intravenosos e insulina como la práctica estándar. Además, es crucial aclarar que, en estos casos, a excepción del paciente 4, tampoco se utilizaron esteroides para tratar la mayoría de las toxicidades inmunitarias causadas por pembrolizumab. Sin embargo, hay que resaltar que se interrumpió el tratamiento con prednisona del paciente 4 luego de 25 días de no observar alguna mejoría en el control glucémico. En contraste con el caso presentado frente a estos 6 casos de la literatura, 4/6 pacientes fueron sometidos previamente a ipilimumab antes de cambiar al tratamiento con pembrolizumab, debido a falta de respuesta o intolerancia.

De acuerdo con la revisión de la literatura de los pacientes que han presentado este caso similar al nuestro, es posible señalar que este representa el primer caso en Colombia. A manera de comparación, en aquellos pacientes que fueron tratados con ipilimumab, se intensificó la toxicidad inmunitaria de pembrolizumab y podría haber sido en estos casos un factor agravante en la precipitación de la diabetes autoinmune (1). Otro punto para tomar en consideración en la presentación de CAD, en la mayoría de los casos 5/7 pacientes, lo cual puede ser un indicativo de que la patofisiología de esta toxicidad inmunitaria tiende a ser aguda. Esta condición también puede alertar al médico sobre este efecto adverso. Lo anterior, se presenta como la oportunidad de ser manejado a manera de protocolo el tratar con los pacientes la posibilidad de aparición de estos afectos, sea CAD o DM, pues se deben discutir como punto clave antes de iniciar la inmunoterapia. Por lo tanto, en esto radica la importancia de llevar a cabo estudios futuros en relación con la predisposición de factores de riesgo asociados con pembrolizumab. 
Tabla 2. Datos demográficos de los pacientes, neoplasias malignas asociadas, presentación clínica y resultado.

\begin{tabular}{|c|c|c|c|c|c|c|c|c|c|}
\hline $\begin{array}{c}{ }^{*} \text { Casos } \\
\text { reportados }\end{array}$ & Paciente & $\begin{array}{l}\text { Edad/ } \\
\text { sexo }\end{array}$ & Diagnóstico & $\begin{array}{l}\text { Otras } \\
\text { inmuno- } \\
\text { terapias }\end{array}$ & $\begin{array}{l}\text { Quimio- } \\
\text { terapia }\end{array}$ & $\begin{array}{l}\text { Presen- } \\
\text { tación }\end{array}$ & $\begin{array}{l}\text { Número } \\
\text { de dosis }\end{array}$ & Anticuerpo & Resultado \\
\hline $\begin{array}{l}\text { Humayun } \\
\text { \& Poole } \\
\text { (18) }\end{array}$ & 1 & $55 / \mathrm{M}$ & Melanoma & Ipilimumab & Dacarbazina & CAD & 9 ciclos & Negativo & $\begin{array}{c}\text { Con } \\
\text { insulina }\end{array}$ \\
\hline $\begin{array}{l}\text { Gaudy et } \\
\text { al. (4) }\end{array}$ & 2 & $44 / F$ & Melanoma & Ipilimumab & No se indica & CAD & 2 ciclos & Negativo & $\begin{array}{c}\text { Con } \\
\text { insulina }\end{array}$ \\
\hline $\begin{array}{l}\text { Hughes et } \\
\text { al. (19) }\end{array}$ & 3 & $64 / F$ & Melanoma & N/A & N/A & CAD & $\begin{array}{l}\text { No se } \\
\text { indica }\end{array}$ & Negativo & $\begin{array}{c}\text { No } \\
\text { conocido }\end{array}$ \\
\hline $\begin{array}{l}\text { Chae et al. } \\
\text { (8) }\end{array}$ & 4 & $76 / \mathrm{M}$ & NSCLC & $\mathrm{N} / \mathrm{A}$ & $\begin{array}{c}\text { Carboplatino } \\
\text { y paclitaxel }\end{array}$ & DM & 2 ciclos & $\begin{array}{l}\text { Anti-GAD } \\
\text { positivo }\end{array}$ & $\begin{array}{c}\text { Con } \\
\text { insulina }\end{array}$ \\
\hline $\begin{array}{l}\text { Hansen et } \\
\text { al. (10) }\end{array}$ & 5 & $58 / \mathrm{M}$ & Melanoma & Ipilimumab & N/A & DM & 17 ciclos & $\begin{array}{l}\text { Anti-GAD } \\
\text { positivo }\end{array}$ & $\begin{array}{c}\text { Con } \\
\text { insulina }\end{array}$ \\
\hline $\begin{array}{l}\text { Martin- } \\
\text { Liberal et } \\
\text { al. (20) }\end{array}$ & 6 & $54 / F$ & Melanoma & Ipilimumab & $\mathrm{N} / \mathrm{A}$ & CAD & 3 ciclos & $\begin{array}{l}\text { Anti-GAD } \\
\text { positivo }\end{array}$ & $\begin{array}{c}\text { Con } \\
\text { insulina }\end{array}$ \\
\hline $\begin{array}{l}\text { Nuestro } \\
\text { caso }\end{array}$ & 7 & $54 / F$ & NSLC & N/A & N/A & $\begin{array}{l}\mathrm{CAD} / \\
\mathrm{DM}\end{array}$ & 4 ciclos & $\begin{array}{l}\text { Anti-GAD } \\
\text { positivo }\end{array}$ & $\begin{array}{c}\text { Con } \\
\text { insulina }\end{array}$ \\
\hline
\end{tabular}

F: femenino; M: masculino; N/A: no aplica.

*Los datos de los primeros 6 casos que se presentan en esta tabla fueron tomados de Cheema et al. World J Oncol. 2018;9(1):1-4.

\section{Conclusión}

Debido a que la diabetes tipo 1 asociada al tratamiento con pembrolizumab es una complicación infrecuente, es necesario establecer protocolos de manejo y posibles marcadores que pronostiquen cuáles pacientes puedan desarrollar estas complicaciones. Esto con el fin de discutir previamente con los pacientes los posibles efectos adversos que pueda presentarse. Además, con el advenimiento de nuevas indica- ciones de la inmunoterapia, se incrementará la presentación de estas entidades y, por lo tanto, es fundamental aprender a reconocerlas dada la mortalidad y la morbilidad asociadas. De igual forma, el estándar del manejo y la monitorización en esta condición no están establecidos, por lo que se reitera en la importancia de formular recomendaciones para la monitorización, el seguimiento y el manejo de estas complicaciones poco frecuentes.

\section{Referencias}

1. Cheema A, Makadia B, Karwadia T, Bajwa R, Hossain M. Autoimmune diabetes associated with pembrolizumab: A review of published case report. World J Oncol. 2018;9(1):1-4. doi: 10.14740/wjon1085w

2. Abdel-Wahab N, Shah M, Suarez-Almazor ME. Adverse events associated with immune checkpoint blockade in patients with cancer: a systematic review of case reports. PLoS One. 2016;11(7):e0160221. doi: 10.1371/ journal.pone.0160221

3. Faghfuri E, Faramarzi MA, Nikfar S, Abdollahi M. Nivolumab and pembrolizumab as immune-modulating monoclonal antibodies targeting the PD-1 receptor to treat melanoma. Expert Rev Anticancer Ther. 2015;15(9):98193. doi: $10.1586 / 14737140.2015 .1074862$

4. Smith-Cohn, M. A., Gill, D., Voorhies, B. N., Agarwal, N., \& Garrido-Laguna, I. (2017). Case report: pembrolizumab-induced Type 1 diabetes in a patient with metastatic cholangiocarcinoma. Immunotherapy, 9(10), 797-804.
5. Gaudy C, Clevy C, Monestier S, Dubois N, Preau Y, Mallet S, et al. Anti-PD1 pembrolizumab can induce exceptional fulminant type 1 diabetes. Diabetes Care. 2015;38(11):e182-3. doi: 10.2337/dc15-1331

6. Maamari, J., Yeung, S. C. J., \& Chaftari, P. S. (2019). Diabetic ketoacidosis in duced by a single dose of pembrolizumab. The American journal of emergency medicine, 37(2), 376-e1.

7. Ansari MJ, Salama AD, Chitnis T, Smith RN, Yagita H, Akiba H, et al. The programmed death-1 (PD-1) pathway regulates autoimmune diabetes in nonobese diabetic (NOD) mice. J Exp Med. 2003;198(1):63-9. doi: 10.1084/ jem.20022125

8. Chae YK, Chiec L, Mohindra N, Gentzler R, Patel J, Giles F. A case of pembrolizumab-induced type- 1 diabetes mellitus and discussion of immune checkpoint inhibitor-induced type 1 diabetes. Cancer Immunol Immunother. 2017;66(1):25-32. doi: 10.1007/s00262-016-1913-7 
9. Aleksova J, Lau P, Soldatos G, McArthur G. Glucocorticoids did not reverse type 1 diabetes mellitus secondary to pembrolizumab in a patient with metastatic melanoma. BMJ Case Rep. 2016;2016:bcr2016217454. doi: 10,11135/bcr-2016-217454

10. Hansen E, Sahasrabudhe D, Sievert L. A case report of insulin-dependent diabetes as immune-related toxicity of pembrolizumab: presentation, management and outcome. Cancer Immunol Immunother. 2016;65(6):765-7. doi: $10.1007 / s 00262-016-1835-4$

11. Sanlorenzo M, Vujic I, Daud A, Algazi A, Gubens M, Alcántara S, et al. Pembrolizumab Cutaneous Adverse Events and Their Association With Disease Progression. JAMA. 2015;151(11):1206-12. doi: 10.1001/jamadermatol.2015.1916

12. Baxi S, Yang A, Gennarelli R, Khan N, Wang Z, Boyce L, et al. Immune-related adverse events for anti-PD-1 and anti-PD-L1 drugs: systematic review and meta-analysis. BMJ. 2018;360:k793. doi: 10.1136/bmj.k793

13. Shamy T, Aguasvivas M, Serhan M, Fojas M. Diabetic Ketoacidosis Trig gered by Pembrolizumab in a Patient with Bladder Cancer. Diabetes 2018;67(Supplement 1). doi: 10.2337/db18-219-LB

14. Manrique C, Silva D. Pancreatic beta cells apoptosis in type 1 diabetes. Rev Fac Med. 2006;54(3):181-90.
15. Alarcon-Casas L, VargasR, Batacchi Z, Hirsch I. Progression to insulin dependence post-treatment with immune checkpoint inhibitors in pre-existing type 2 diabetes. AACE Clin Case Rep. 2017;3(2):e153-7.

16. Wang P, Chen Y, Song S, Wang t, Ji W, Li S, et al. Immune-Related Adverse Events Associated with Anti-PD-1/PD-L1 Treatment for Malignancies: A Meta-Analysis. Front Pharmacol. 2017;8:730. doi: 10.3389/ fphar.2017.00730

17. Kapke J, Shaheen Z, Kilari D, Knudson P, Wong S. Immune Checkpoint Inhibitor-Associated Type 1 Diabetes mellitus: Case Series, Review of the Literature, and Optimal Management. Case Rep Oncol. 2017;10(3):897909. doi: $10.1159 / 000480634$

18. Humayun MA, Poole R. A case of multiple immune toxicities from Ipilimumab and pembrolizumab treatment. Hormones. 2016;15(2):303-6.

19. Hughes J, Vudattu N, Sznol M, Gettinger S, Kluger H, Lupsa B, et al. Precipitation of autoimmune diabetes with anti-PD-1 immunotherapy. Diabetes Care. 2015;38(4):e55-e57. doi: 10.2337/dc14-2349

20. Martin-Liberal J, Furness AJ, Joshi K, Peggs KS, Quezada SA, Larkin J. Antiprogrammed cell death-1 therapy and insulin-dependent diabetes: a case report. Cancer Immunology, Immunotherapy. 2015;64(6):765-7. 
